\title{
Eye movement analysis and cognitive processing: detecting indicators of conversion to Alzheimer's disease
}

\section{Marta LG Freitas Pereira Marina von Zuben A Camargo Ivan Aprahamian Orestes $\vee$ Forlenza}

Laboratory of Neuroscience (LIM-27), Department and Institute of Psychiatry, Faculty of Medicine, University of São Paulo, São Paulo, SP, Brazil
Correspondence: Marta LG Freitas Pereira

Laboratory of Neuroscience (LIM-27), Department and Institute of Psychiatry, Faculty of Medicine, University of São Paulo, Rua Dr Ovídio Pires de Campos 785, 05403-0 I0, São Paulo, SP, Brazil Email martafreitaspereira@gmail.com
This article was published in the following Dove Press journal:

Neuropsychiatric Disease and Treatment

9 July 2014

Number of times this article has been viewed

\begin{abstract}
A great amount of research has been developed around the early cognitive impairments that best predict the onset of Alzheimer's disease (AD). Given that mild cognitive impairment (MCI) is no longer considered to be an intermediate state between normal aging and $\mathrm{AD}$, new paths have been traced to acquire further knowledge about this condition and its subtypes, and to determine which of them have a higher risk of conversion to AD. It is now known that other deficits besides episodic and semantic memory impairments may be present in the early stages of $\mathrm{AD}$, such as visuospatial and executive function deficits. Furthermore, recent investigations have proven that the hippocampus and the medial temporal lobe structures are not only involved in memory functioning, but also in visual processes. These early changes in memory, visual, and executive processes may also be detected with the study of eye movement patterns in pathological conditions like MCI and AD. In the present review, we attempt to explore the existing literature concerning these patterns of oculomotor changes and how these changes are related to the early signs of AD. In particular, we argue that deficits in visual short-term memory, specifically in iconic memory, attention processes, and inhibitory control, may be found through the analysis of eye movement patterns, and we discuss how they might help to predict the progression from MCI to AD. We add that the study of eye movement patterns in these conditions, in combination with neuroimaging techniques and appropriate neuropsychological tasks based on rigorous concepts derived from cognitive psychology, may highlight the early presence of cognitive impairments in the course of the disease.
\end{abstract}

Keywords: Alzheimer's disease, mild cognitive impairment, eye movement patterns, visuospatial abilities, visual memory, executive functions

\section{Introduction}

A great deal of interest has been generated regarding the best predictors of Alzheimer's disease (AD). However, subjects frequently present with considerable amounts of cognitive deficits before the establishment of the diagnosis. This initial stage of the disease is now called mild cognitive impairment (MCI). This condition has brought a lot of relevant information about the nature of the deficits that occur between normal aging and AD. Currently, researchers have been arguing about its clinical validity as a unitary diagnosis in itself, rather than acting as an intermediate phase in the progression to dementia. ${ }^{1,2}$

However, this debate has drawn attention to a very relevant issue: which early deficits best predict the onset of $\mathrm{AD}$, besides the well-known episodic and semantic memory impairments. ${ }^{3}$ Given the growing evidence showing that visual processing ${ }^{4}$ and executive functions ${ }^{5}$ may also be impaired early in the course of the disease, several researchers 
have been focusing their attention on changes in eye movement patterns as a measure of these impairments. ${ }^{6-8}$

In the present review, it is not our aim to extensively expose all of the existing studies that have been conducted around the changes in eye movement patterns that precede significant deficits in AD pathology, since this would be an impossible goal to achieve. Instead, we will try to very briefly present some of the most relevant investigations around this topic, critically analyzing the methodologies and behavioral tasks used in the referred studies. More specifically, we will try to identify and discuss some theoretical frameworks surrounding cognitive deficits that might be underidentified as early signs of AD - namely, visual and visuospatial impairments, different subtypes of memory impairments, as well as executive control deficits. Also, we will synthesize which eye movement pattern changes might be associated with these deficits in $\mathrm{MCI} / \mathrm{AD}$ patients, and which might need further investigation.

\section{Mild cognitive impairment: still an intermediate state between normal aging and Alzheimer's disease?}

Researchers have been trying to better understand the changes involved in the process of aging, specifically focusing on the cognitive differences between normal and pathological aging. ${ }^{4,9} \mathrm{MCI}$ is generally associated with aging processes, and it is thought to be an intermediate state between normal aging and dementia, thus increasing the risk of progression to $\mathrm{AD} \cdot{ }^{10,11}$ However, not all aging processes lead to cognitive declines, and the pattern of cognitive decline may vary across individuals. MCI is currently regarded as a complex condition that still needs further investigation to better define it, and to develop a rigorous understanding about its clinical utility. ${ }^{1,2}$ The recent subclassification of MCI according to its cognitive features seems to be an effort to set some boundaries regarding the heterogeneity around this concept. ${ }^{10-12}$ Petersen $^{11}$ suggested that MCI be subdivided into two variants: amnestic MCI (with clinically significant memory impairment); and nonamnestic MCI (with significant impairment in functions other than memory, such as attention, executive processing, language, or visuospatial skills). In addition, individuals must present very little functional compromise in daily activities or with insufficient severity. ${ }^{1,9}$

There are currently several means for predicting the progression of MCI, such as structural magnetic resonance imaging (MRI), ${ }^{13,14}$ functional imaging techniques ([18]Ffluorodeoxyglucose positron emission tomography), ${ }^{15}$ analysis of the biomarkers in cerebrospinal fluid, ${ }^{16}$ and the use of molecular imaging. ${ }^{17}$
However, this classification is not widely accepted among the research community, and it is considered to be too restrictive to subdiagnose the MCI population. ${ }^{18}$ In fact, previous research has reported the differences between clinical versus population-based MCI studies, where the clinical classification appears to be more well defined, and the progression of the disease is more uniform than in the population-based studies. This might be due to the fact that people followed in clinical settings probably show a different level of impairment because they actively seek help. ${ }^{19}$ Given this debate, assessing the risk of disease progression is not an easy task, and it has been shown to be influenced by the definition and subtype of MCI, as well as the setting. However, as far as we know, more recent data suggest that there is a rate of progression ranging between $6.8 \%$ (community studies) and $8.1 \%$ (clinical settings). ${ }^{20}$ Other findings report a more significant association between amnestic (a)MCI groups and the progression to AD. ${ }^{5,21}$ In a recent meta-analysis conducted by Mitchel and Shiri-Feshki, ${ }^{20}$ the authors found a higher progression rate for multi-domain MCI and aMCI than non-memory MCI, and concluded that the definition of MCI is still heterogeneous, which undeniably affects its diagnosis and clinical practice.

It is now clear that MCI can no longer be assumed to be a simple transitional state between normal aging and the development of dementia, especially when different subtypes of MCI are yet to be fully defined. Increasing evidence suggests that early deterioration of cognitive domains other than episodic memory is possibly associated with the conversion to $\mathrm{AD}$, given that this type of memory impairment may not be sensitive to all of the early manifestations of the pathology. Recent studies have reported changes in visuospatial abilities $^{4}$ and in executive functions ${ }^{5,22,23}$ before establishing a clinical diagnosis.

In sum, a vast number of studies have traditionally considered episodic and semantic memory changes as the most significant early impairments of $\mathrm{AD}$; however, a growing number of findings have been suggesting that visuospatial and executive deficits may appear earlier in time, and can even precede the typical manifestations of memory impairments associated with MCI and AD, ${ }^{4,5,22,23}$ proving that the accurate evaluation of these domains can represent a promising approach for identifying the predictive markers of AD.

\section{Eye movement patterns as a measure of cognitive impairment}

Eye movement behavior can be a powerful tool to explore higher-order cognitive control processes (Figure 1). It has the potential to sensitively assess disease progression and the severity of cognitive impairment. For several years, 

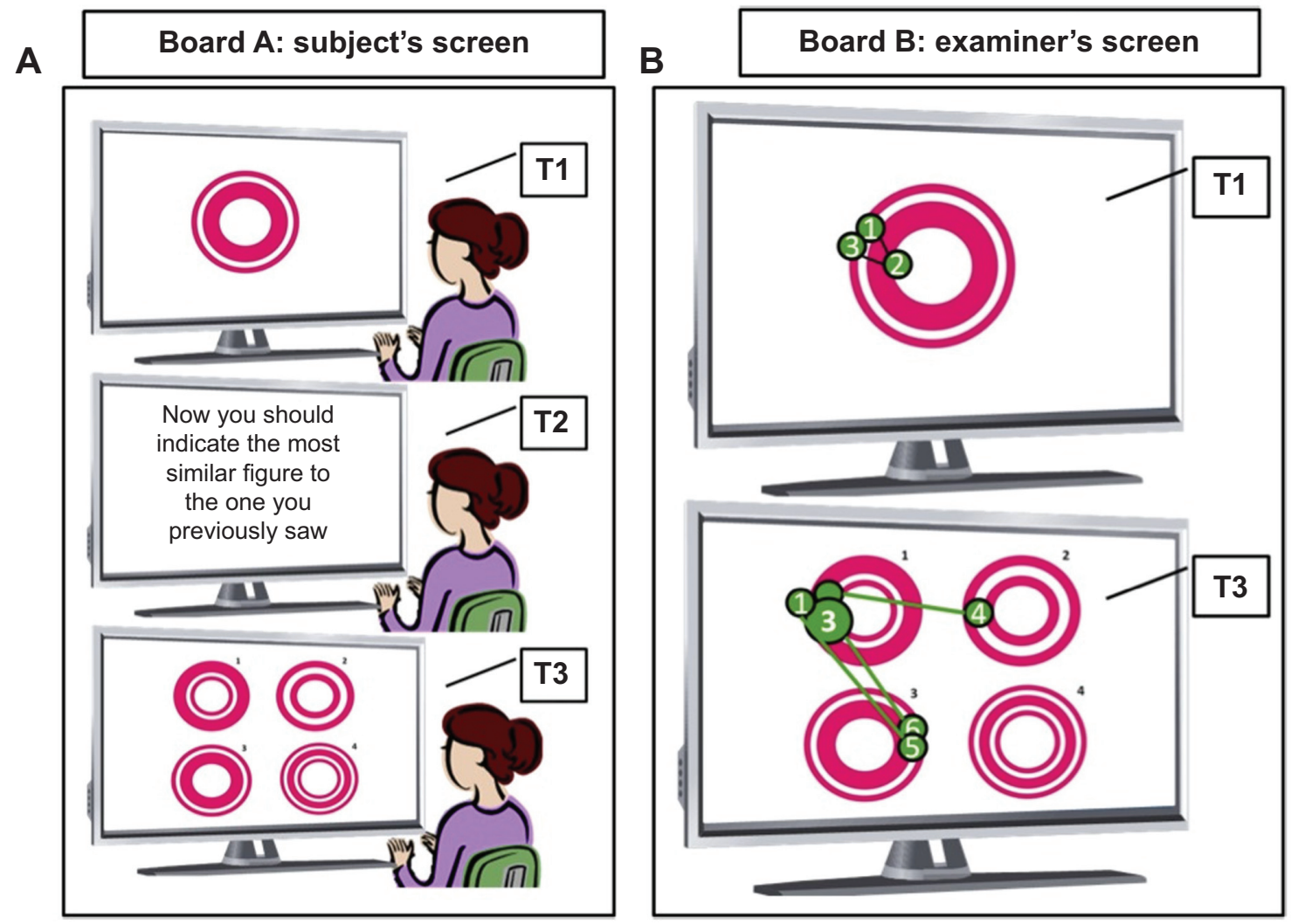

Figure I Computerized task used to measure cognitive performance with an eye-tracker device.

Notes: The figure illustrates a task used to measure cognitive performance using an eye-tracker device. TI, T2, and T3 represent three successive movements during the same task. (A) The screen used by the subject during the test. Before he or she begins, the subject is oriented to pay attention to all the images and instructions presented on the screen. At TI, the first stimulus is presented for a limited period of time, longer or shorter depending on the figure's complexity. At T2, the subject should read the instruction as many times as necessary for him or her to understand what is being asked. When he or she feels ready to accomplish the task, he or she presses any key from the keyboard. At T3, the third screen is presented with the recognition task. In this case, the subject should verbally indicate the figure that perfectly matches the one previously seen, and immediately press a key to continue the test, moving on to the next stimulus. (B) The screen containing representations of the eye movement behavior during test phases $\mathrm{TI}$ and $\mathrm{T} 3$. Each green numbered circle represents one eye fixation at that specific part of the screen. The size of the green circle is determined by the duration of fixation: the bigger the circle, the more time the subject spent on that fixation point. The number on each circle represents the sequence of eye fixations.

researchers have been trying to identify which kind of eye movements can highlight some of the cognitive deficits that present in the early course of AD.

Thus far, a vast majority of studies have been focusing on verbally-mediated memory disorders as the earliest predictors of AD - specifically, episodic and semantic memory impairments. ${ }^{24,25}$ Nonetheless, recent work suggests that other deficits, such as visual behavior impairments, can accurately predict $\mathrm{AD}$ and contribute to the differential diagnosis of other pathological conditions like multiple sclerosis and Parkinson's disease ${ }^{8,26-29}$ Moreover, other findings indicate that oculomotor assessment may aid in the diagnosis of frontotemporal lobar degeneration and related disorders. ${ }^{30}$ It may also differentiate $\mathrm{AD}$ patients from those with semantic dementia, ${ }^{31}$ and it has proved to be a valuable method for indexing memory function in special populations, ${ }^{7,32,33}$ such as normal aging populations and those with psychiatric disorders as well, ${ }^{7,34}$ given that eye movements can be acquired without explicit reports or other overt responses.
Some studies have been showing that patients with $\mathrm{AD}$ are less able to process color information..$^{29,35-41}$ These findings, combined with the increasing numbers of neurofibrillary tangles present in the visual association cortex, particularly in temporal and parietal lobes, ${ }^{42-46}$ may explain the difference between AD and control subjects' performance on different tasks that involve features such as color perception, ${ }^{36,41}$ stereoacuity, ${ }^{35}$ perceptual organization, contrast sensitivity, ${ }^{35,37,38,47,48}$ backward masking, ${ }^{49}$ spatial reasoning, ${ }^{48,50}$ or face and object recognition. ${ }^{35}$

Predictive pursuit can be also observed through the analysis of eye movements. Efficient object pursuit requires appropriate target selection and predictive compensation for inherent processing delays. Prediction depends on the expectation of future object motion, the storage of motion information, and the use of extraretinal mechanisms, in addition to visual feedback. Fukushima et a ${ }^{51}$ reviewed different studies conducted with both humans and primates, and they presented behavioral evidence of how cognitive processes are 
involved in predictive pursuit in normal humans; they then described neuronal responses in monkeys and behavioral responses in patients. More specifically, the authors explained how these cognitive controls occur, by examining the neural substrate of working memory and movement preparation for predictive pursuit through a memory-based task in macaque monkeys. This kind of task, when applied to patients with Parkinson's disease, revealed deficits in movement preparation, but not in working memory. However, patients with frontocortical or cerebellar dysfunction seemed to exhibit working memory impairments.

In fact, eye movement impairments may also have some diagnostic value in the early identification of MCI. ${ }^{6-8,29,52}$ More specifically, certain deficits in visual functions may be present in MCI patients who do not present objective visual complaints. ${ }^{29}$ In his research, Rizzo et al ${ }^{29}$ tested 43 patients with mild severity $\mathrm{AD}$ and 22 nondemented subjects with a battery of tests designed to assess basic and higher-order visual perception and cognition. Their results suggested that visual dysfunction in AD (static spatial contrast sensitivity, visual attention, shape-from-motion, color, visuospatial construction, and visual memory) is highly correlated with several impairments in other cognitive domains.

Several characteristics associated with eye movements such as fixation duration, refixations, saccade orientation, and pupil diameter - can help to improve the classification accuracy of cognitive impairment. In Lagun et al's work, ${ }^{8}$ the authors used classification algorithms (dispersion-based fixation detection algorithms) combined with machine learning methods to more accurately distinguish between healthy (30 normal age-matched controls) and impaired subjects (ten MCI and $20 \mathrm{AD}$ patients). They found that when using extended eye movement representation features (novelty preference, saccade orientation, refixations, and fixation duration), it was possible to distinguish normal controls from MCI subjects with $87 \%$ accuracy, 97\% sensitivity, and $77 \%$ specificity of cognitive impairment detection based on eye-tracking data. Lagun et al's work ${ }^{8}$ also elucidated the possibility of potentially detecting hippocampal or other structural damages not yet apparent via functional MRI (fMRI) imaging. This study offers the advantage of distinguishing - with high accuracy rates - patients with MCI from normal controls.

Since Jones et al's ${ }^{53}$ first study concerning eye movement behavior impairments in four AD patients was performed, several studies have been conducted revealing deficits such as saccadic intrusions, defects in fixation and smooth saccadic eye movements, ${ }^{54}$ slow pursuit movements, ${ }^{55}$ and increased catch-up saccades. ${ }^{56}$ More recently, Mosimann et al ${ }^{57}$ have run an experiment about visual exploration behavior during a clock-reading task adapted to a computer screen, where eye movement measurements were registered with an eye tracking device. The results showed that patients with probable AD (number =24) exhibited less focused exploration movements, with fewer fixations inside the region of interest, and with a significant delay in time before the first fixation occurred inside the region of interest. The eye movement pattern revealed longer fixations and smaller saccade amplitudes. These patients exhibited a different pattern of exploration, which the authors related to parietal dysfunction. However, these changes were not due to global cognitive impairments, but rather to impaired clock reading. This kind of study shows, however, the importance of eye movement behaviors in everyday life and its relation with "automatic" tasks.

Conversely, eye movement behaviors in MCI or early AD patients have not been studied extensively. Research has demonstrated that the patients show altered visual search strategies and eye movement behaviors, with deficits in smooth pursuit eye movements, an increased number of saccades, as well as increased attentional deficits and eye blinks. ${ }^{58}$ Increased latencies in a reflexive saccade overlap task have also been found in MCI patients when compared to age-matched normal controls. ${ }^{59}$ Other findings have shown that alterations in oculomotor exploration may also reveal other cognitive impairments in the MCI groups, namely in executive functioning. MCI subjects may also exhibit altered saccadic inhibition when compared to healthy elderly subjects. In a saccadic inhibition study run by Alichniewicz et $\mathrm{al}^{5}$ the authors have combined eye-tracking methodology with fMRI data and have found that aMCI subjects revealed decreased activation in the frontal eye fields when compared to healthy elderly persons during antisaccade performance, which can be interpreted as a decline in inhibitory functioning in early manifestations of AD.

In sum, it is possible nowadays to identify altered patterns of oculomotor behavior in a wide variety of pathological conditions, and to help establish differential diagnoses in clinical settings. There is evidence of the presence of oculomotor deficits in MCI patients that reveal different types of cognitive impairments (for example, impairments in memory and executive functioning); however, these studies have restricted populations and are not extended in time. It would be interesting if future investigations could develop more longitudinal studies conducted with larger populations in order to verify how these changes in visual processing 
develop with time, and to determine whether they really have some predictive strength in AD.

\section{Eye movement and memory impairments in MCI/AD}

It is important to better understand how eye movement behavior is linked to early manifestations of memory impairments in dementia. In fact, eye movements have been shown to reveal the influence of different types of memory (for example, item memory and relational memory), permitting us to clarify several issues about distinct memory systems. $^{7,34,60,61}$

Some authors have been trying to use behavioral tasks that can reveal these visual deficits without needing to produce almost any verbal responses, and which are suitable for testing subjects with cognitive impairments, such as AD patients. ${ }^{8,52}$ One of the tasks known for its high sensitivity to early memory impairments is the visual-paired comparison task, which is generally used to assess memory recognition by focusing on the tendency that subjects will explore novel items in more detail. In this task, an item is briefly presented, and after a delayed period of time, the previously seen item is presented again, side by side, with a novel item. The amount of time spent viewing this novel item when compared to the old item is then measured, and if a preference for the new item is found, it can be assumed that the subject has a memory for the familiar, less-viewed item. This task was able to detect impaired declarative memory in humans and in nonhuman primates, who had little detectable damage in the medial temporal lobe (MTL) memory system, as they showed equal amounts of time spent on viewing both the novel stimulus and the familiar stimuli. ${ }^{6,34}$ According to the authors, the influence of different cognitive deficits on these results still needs to be investigated further, although the assumption that these eye movement patterns are related to impaired memory remains compelling. The posterior memory recognition task sufficiently challenged the memory system so that MCI subjects no longer remembered which image they had previously seen. ${ }^{6,8}$ Amnesic patients exhibited a decrease in the amount of time spent viewing repeated items, as compared to novel ones, like faces and scenes. ${ }^{60}$

Some other tasks involve mechanisms associated with binding (ie, linking) different features across different dimensions (ie, color, shape, or location) or visual streams (ie, ventral or dorsal). The differences observed across studies may arise from the different demands of these tasks to develop effective connectivity across or within these feature dimensions. ${ }^{62-64}$ Studies have shown that AD patients perform poorly on short-term memory tasks involving shape-color binding. These results have been extended to suggest that specific impairments in binding the information stored in memory differentiates AD patients' performance from that of patients with major depression. ${ }^{65}$

In fact, the literature has revealed that visual search strategies are not random; rather, they are guided by two factors: the physical properties of the elements presented in a specific scene; and our previous experience with and knowledge about that particular situation (which is provided by episodic and semantic memory systems). ${ }^{7}$ The visual memory representation derived from the previewed scene facilitates a later search through that scene. Therefore, scene memory representation in guiding visual search is not limited to spatial layout, but it also preserves information about the form and/or identity of the objects occupying those locations. ${ }^{61,66}$ Also, the hippocampus is needed to form these allocentric representations from previously seen material. ${ }^{67}$

Troyer et a ${ }^{68}$ compared 29 subjects diagnosed with aMCI to 30 age-matched controls on object-location and symbolsymbol recall tasks. Although item recall was impaired in the aMCI group relative to controls, the associative recall proved to be even more impaired in the aMCI group. Impairments in this ability to recollect, in an integrative way, associative information from memory are often associated with early neuroanatomical changes, namely in the hippocampus and entorhinal cortex. In fact, these measures of associative recall were the best group discriminators, with scores of $76 \%$ sensitivity and $90 \%$ specificity for symbol-symbol recall and $86 \%$ sensitivity and $97 \%$ specificity for objectlocation recall. ${ }^{68}$ The authors concluded, then, that associative memory deficits can be an early cognitive sign of AD.

Patients with $\mathrm{AD}$ and asymptomatic carriers of the E280A single presenilin-1 mutation have also been studied using tasks involving memory recognition of shape, color, and shape-color binding, and they performed significantly worse than healthy controls in the feature-binding condition alone. ${ }^{65,69}$ Standard neuropsychological tasks revealed no significant differences between asymptomatic carriers and healthy controls. Statistical analyses confirmed the greater sensitivity and specificity of binding tests than other traditional neuropsychological measures for patients with $\mathrm{AD}$, and most notably for asymptomatic carriers of the mutation, which suggests that visual short-term memory (VSTM) binding deficits may be a preclinical marker for familial AD. ${ }^{65,69}$ Patients with AD have impairments not only in VSTM, but also in more high-order visual perceptive functions, such as divided attention, selective attention, visual memory ${ }^{29}$ 
and also in semantic interference. ${ }^{70}$ However, not all low-level perceptual functions remain intact in AD. It is known that contrast sensitivity deeply affects other high-level visual cognition tasks, such as recognition tasks. ${ }^{29}$ This perceptual impairment has proven to deeply affect other high-level visual cognition tasks, such as recognition tasks. ${ }^{35}$ It would be interesting if future investigations could try to understand whether other low-level perceptual features could be also impaired in the early stages of AD, as they can be easier rehabilitated and will provide a better quality of life for these subjects.

Subsequent research on VSTM has shown AD/MCIrelated deficits in at least three important stages. First, iconic memory decays much faster in patients with MCI compared to healthy controls. ${ }^{71}$ Second, consolidation of information in VSTM may be impaired in AD patients. Third, VSTM binding is changed in patients with AD. More specifically, AD patients seem to have a specific deficit in how features of objects in VSTM are bound together. ${ }^{65}$ The consolidation process from iconic memory to VSTM seems to remain intact for MCI patients, but it might become corrupted once MCI patients progress into AD. Overall, the deficits in iconic memory described in MCI patients might be considered high risk factors for $\mathrm{AD}$ conversion. This conclusion was corroborated by Lu et $\mathrm{al}^{71}$ who compared observers with MCI to young and older control subjects, and found that iconic memory decayed much faster for the MCI group than for the normal control groups (young or old).

In an attempt to determine which modality - VSTM or visuospatial short-term memory (VSSTM) - decays faster in the early phases of $\mathrm{AD}$, a recent study ${ }^{72}$ compared the performance of MCI and AD groups and concluded that VSTM and VSSTM were significantly impaired in the MCI and AD groups as compared to healthy age-matched controls, with VSSTM scores being worse than VSTM scores. These results seem to indicate that visual deficits might be related (or be secondary) to impairments in other cognitive functions, such as attentional and executive nature deficits.

Previous investigations concerning the theory of multiple memory systems ${ }^{66,73}$ have emphasized the role of the MTL (and, specifically, the hippocampus) in the conscious recollection or conscious awareness of prior experiences. These studies have highlighted the MTL's importance in episodic and semantic memory. They also placed emphasis on its implications in the recovery of perceptual details and/or of more complex information. ${ }^{74}$

Additional studies ${ }^{75-77}$ have indicated that awareness is not always necessarily required in memory expression in the eye movement patterns. The findings revealed that hippocampal amnesia has a significant impact on relational memory, while sparing item memory, regardless of issues of conscious access. Hence, recent findings have reported that the use of eye movement paradigms provided critical evidence in favor of a relational memory account over an explicit memory account with respect to impairments in hippocampal amnesia., ${ }^{7,60}$

In conclusion, there has been growing evidence that some behavioral tasks, which were built to detect covert memory impairments through the analysis of eye movements, can detect $\mathrm{AD}$ early in the course of the disease, while indicating possible changes in hippocampal functioning. Also, visual memory binding deficits may be a preclinical marker, as they are present even in MCI patients. More specifically, iconic memory seems to decay faster in this group, and it might be considered a high risk factor of AD conversion.

\section{Eye movement and attention/ executive impairments in MCI/AD}

As previously mentioned, recent findings have been pointing out that other deficits - besides those in episodic and semantic memory - might appear early in AD. Specifically, alterations in some types of attention and inhibitory control have been found, with some consistency in patients diagnosed with $\mathrm{AD} / \mathrm{MCI}$. It is important, then, to clarify some ideas around these concepts.

Although episodic memory has been widely considered among one of the first deficits in $\mathrm{AD}$, some studies have examined attention and visual attention in the early stages of the disease. ${ }^{78}$ Early studies conducted with probable AD patients using eye movement records revealed significant impairments in tasks that required an active shift of focal attention. Scinto et $\mathrm{al}^{79}$ showed that these patients exhibited less accuracy and longer saccade latencies when they were asked to fixate on a target appearing randomly to the right or left of a central point, and to direct their attention to a target appearing randomly in one of four peripheral locations. Furthermore, in the second task, the authors observed an increase of deficits, as task demands were made more difficult. Specifically, a failure to accurately shift one's attention resulted from perseveration errors and a lack of persistence. The overall conclusion suggested that the deficits in attention might play a role as an early marker of $\mathrm{AD}$, and that eye movement data can provide useful and accurate information if combined with appropriate tasks that can manage different levels of attention demands.

However, although a previous investigation has focused on visual attention deficits as a preclinical marker of $\mathrm{AD}$, very 
few studies have approached visual attention as a multidomain concept, with some aspects being more vulnerable than others in different pathological conditions. Okonkwo et a ${ }^{80}$ investigated potential visual attention deficits by comparing performances between a group of 51 aMCI patients and 58 normal controls. Using the Useful Field of View test, the authors tried to assess simple, divided, and selective attention in both groups, and the results showed that divided attention was the most impaired in a hierarchical pyramid of attentional deficits, with $53 \%$ of the MCI patients being impaired. Despite the relatively large number of individuals tested in this investigation, the majority of the research concerning attentional deficits in MCI/AD conditions have restricted and limited sample sizes, which inevitably create some constraints in the an early detection of these deficits.

Lonie et al ${ }^{81}$ examined group differences between aMCI patients, early $\mathrm{AD}$ patients, depressive controls, and healthy elderly controls in a dual task performance. They concluded that there were no group differences in this kind of task; however, aMCI and early $\mathrm{AD}$ groups seemed to have marked difficulties when dividing their attention between tasks, such as on part B of the Trail Making Test. The authors suggested that a more taxing dual task measure might be more sensitive to early divided attention changes in $\mathrm{AD}$.

Perry and Hodges ${ }^{82}$ tested nine MCI subjects and nine controls on a paradigm that quantified both the attentional blink and top-down control of attention in a visual search task. Subjects were asked to identify certain stimuli that were presented sequentially, and the observed detrimental effect in identifying a second stimulus, after identifying the first one, served as a measure of the attentional blink. When asked to identify only one of the two stimuli, the subjects' ability to ignore the first stimulus served as a function of their topdown attentional control. Results showed that MCI subjects had a normal attentional dwell time, but they had impaired top-down attentional control. The authors suggested that these two aspects of visual search are mediated by different neural systems, with top-down processing being connected to the prefrontal cortex and the attentional blink being a function of earlier perceptual processing in the visual cortices. They also added that this more complex, top-down processing can also be a very early sign of AD. ${ }^{82}$

With regards to visuospatial attention strategies in $\mathrm{AD}$, some deficits have been registered - namely, an increased number of fixations and longer duration of fixations. Rösler et al ${ }^{83}$ examined a group of $\mathrm{AD}$ patients, a group of age-matched controls, and another group of young controls on a computerized task, where subjects had to search for a number among a nonlinear array of letters on a large screen. The results indicated that $\mathrm{AD}$ patients made more fixations and exhibited longer fixation times than the other two groups, which delayed target detection. These differences in the visual search patterns between groups were interpreted as deficits in disengaging visuospatial attention, but it was also associated with a prolongation of saccade initiation and inefficiency in planning a search strategy. Other authors have corroborated this failure to disengage and shift attention, especially on novel tasks, where participants have failed to inhibit wellrehearsed strategies (see Perry and Hodges ${ }^{78}$ for a review).

It seems undeniable that these alterations in visuospatial attention strategies need to be further investigated, given that they can provide some clues about other executive function deficits. Concerning inhibitory deficits, a more recent study $^{84}$ has suggested that these kinds of deficits may be explained by direct degenerative effects on inhibitory control. In their study, Crawford et al ${ }^{84}$ tested 18 patients with probable $\mathrm{AD}$ and two control groups (17 young participants and 18 older participants) concerning four saccadic eye movement paradigms: prosaccade; $\mathrm{NO}-\mathrm{GO}$; $\mathrm{GO} / \mathrm{NO}-\mathrm{GO}$; and antisaccade. The results showed fewer error correction saccades in patients with probable $\mathrm{AD}$, and an inability of these patients to correct erroneous saccades. The authors suggested that these failures provided the most reliable oculomotor index of dementia severity, distinguishing them from the normal effects of aging. However, an eventual capacity of early detection of AD does not become clear, in contrast to what had been suggested by Rösler et $\mathrm{al}^{83}$ with the dual task performance.

Despite the fact that there are inhibition deficits in AD, little is known about these kinds of deficits in patients with MCI and its predictive value in dementia processes. Furthermore, some controversy has been raised in the literature concerning the presence of inhibitory deficits in $\mathrm{MCI}$ patients. While some authors ${ }^{85}$ point out that these patients exhibit impairments in inhibitory functions (mainly, reduced resistance to interference and partially impaired goal maintenance), others ${ }^{86}$ claim that these deficits are not relevant in MCI. In a more recent study, Alichniewicz et $\mathrm{al}^{5}$ combined eye tracking and fMRI measures to investigate the neural correlates of oculomotor functions in aMCI patients. The authors pointed out that there was increased activation of the frontal and parietal regions in both prosaccades and antisaccades for the aMCI, healthy elderly, and younger groups. Nonetheless, the aMCI group exhibited significantly decreased activation in the frontal eye field region, bilaterally, in comparison to the healthy elderly group during the antisaccade task 
performance. We can thus argue that the decline in inhibition functioning and the corresponding disruption in frontal lobe activity in aMCI might be a sensitive measure of the early manifestation of AD.

Further research is still needed regarding the nature of inhibitory impairments in AD/MCI patients, but the evidence seems to indicate that these deficits might be one of the first signs of the presence of executive function alterations in these patients. It would be relevant if, in the future, researchers could combine eye movement methodology with neuroimaging techniques in order to explore these impairments with greater accuracy.

\section{Memory and attention expression in eye movement behavior: two systems operating together?}

It is not always easy to tease apart the effects of attention and memory on visual processing. Memory retrieval is usually associated with activation of the parietal cortex, which is also implicated in the attentional system. Some findings have provided evidence that MTL structures may play a perceptive role on the memory system, namely in the perirhinal cortex ${ }^{87}$ when discriminating between different conditions of feature ambiguity. Therefore, researchers are still looking to develop a better understanding of the existing relationship between these different cognitive domains on AD pathology.

Some authors have also suggested that the inferior parietal lobe mediates the automatic allocation of attention to retrieved memory contents. Moscovitch ${ }^{66}$ hypothesized that this region is associated with the recovery of information from the hippocampus. Therefore, the inferior parietal lobe is active when attention is allocated by memory contents for vivid memories, rather than for merely familiar ones. The author added that the capture of attention by strong, vivid memories has priority over an overt allocation of attention in perceptual tasks. In addition, Olsen et $\mathrm{al}^{88}$ added that overt shifts of attention through eye movements are associated with higher accuracy of performance in relational visuospatial memory tasks - ie, attention seems to facilitate memory for the relationships between objects. The authors argued that the activation of these parietal lobe regions, which are also known to be involved in visuospatial attention and in oculomotor planning, may reinforce and help to produce stronger relational spatial representations and, consequently, more accurate memory recognition of previously studied items. We can assume, therefore, that there is a combined influence of both attention and memory (namely, visuospatial memory) in the formation of visual representations. However, attentional processes seem to play other roles in the identification of early impairments associated with $\mathrm{AD}$, besides facilitating memory retrieval.

Further, deficits in visual memory recognition in AD/MCI patients can be secondary to impairments in attentional and executive resources, whereas deficits in visuospatial memory recognition appear to be primary, and they reflect a genuine spatial disorder. ${ }^{89}$ Corroborating this theory that visuospatial recognition is a primary impairment is the fact that $\mathrm{AD}$ patients tend to show increased pupil dilation during a visual search for conjunctions rather than features, suggesting increased effort during such conditions. ${ }^{90}$ Scene descriptions from AD subjects with hippocampus/MTL lesions are poorer in detail, especially in spatial coherence, and these descriptions reported rather more isolated features and fragments than an integrated coherent spatial conjunction. ${ }^{67}$

Another study ${ }^{91}$ that tested spatial navigation impairments in different subtypes of MCI confirmed that the aMCI group showed significant spatial navigation impairments in allocentric processing, whereas the nonamnestic and subjective memory complaints groups registered similar values to controls. In addition, the $\mathrm{AD}$ and aMCI multiple domain (aMCImd) groups were impaired in all spatial navigation conditions. The authors thus suggested that spatial navigation impairments in aMCI could be detected at an earlier stage. They added that this deficit could allow for the monitoring of disease development, starting from aMCI to aMCImd and ending in AD. However, further investigations based on the complementary assessment of attentional resources and visuospatial memory are needed to help identify the cognitive origin and neurofunctional bases of visual processing deficits, as shown in MCI and AD patients. This is necessary to understand the staging of the deficits and to determine their predictive value. Moreover, given the lack of consensus around the definition of MCI, more studies are needed to determine which types of MCI show impairments in visuospatial memory and which of them convert to AD.

Considering the impairments in visual processing capacity, Bundesen ${ }^{92}$ developed a theoretical framework that explores the integration of different components of visual information intake, known as the "theory of visual attention". According to this theory, an object can be identified and encoded into VSTM, and the probability of its correct identification is a function of exposure duration. If multiple objects are presented in the visual field, then a competition between multiple objects for selection is established as a race towards VSTM. A subject's performance can vary according to perceptual threshold, iconic memory, processing speed, and 
VSTM. When applied to clinical populations, like MCI and $\mathrm{AD}$ patients, ${ }^{93}$ the theory of visual attention indicates that $\mathrm{MCI}$ shows a rise in perceptual thresholds, while AD is affected in terms of processing speed and VSTM storage capacity. This means that the elevated thresholds are related to impaired preattentive (bottom-up) mechanisms in both AD and MCI, while the allocation of attention to stimuli and the storage of selected items in VSTM seem to be more affected in AD than in MCI patients. In other words, the results revealed deficits in preattentive visual processing in MCI, but only in attentive processing in AD. Moreover, the perceptual thresholds were associated with disease duration, but not with cognitive measure, with the reverse being true for speed and the VSTM parameters. This study suggests that either the early attentional deficits or the later VSTM features affect visual processing in $\mathrm{AD}$ at different stages of the disease, anticipating its predictive power as a distinct marker during the course of MCI, before the appearance of memory impairments. Once again, longitudinal studies would be very relevant in verifying which types of MCI subjects convert to AD. Nevertheless, these findings show that attention and memory are two parts of one continuum of the cognitive changes associated with $\mathrm{AD}$, although the authors suggest that preattentional and attentional deficits might be an early marker of the disease. In addition, it would be interesting to investigate these preattentional alterations with tasks that are especially designed for the study of selective attention processes. Specifically, it would be useful to identify how shifts in attention and inhibitory control changes affect the performance of selective attention. Eye movement methodology, along with neuroimaging techniques, could bring an invaluable contribution to this study.

The overall results highlighted the growing relevance of changes in attention associated with the development of AD. Yet, the predictive role of memory alterations early in the course of the disease is undeniable. Nevertheless, we already have some clues about the other types of memories that can also be affected, such as visual and visuospatial memories, due to the contributions of eye movement studies. In sum, both attention/executive functioning and memory impairments are known to be early cognitive markers, and even though they first seem to influence visual memory performance, more experimental evidence is needed to state whether executive and attentional deficits might occur earlier and influence later visual processing impairments.

\section{Conclusion}

Cross-sectional studies have been conducted assessing AD patients and controls, ${ }^{32,53,55,57,58,79,81,83,84,90,92}$ AD and
MCI patients, ${ }^{8,52,59}$ MCI patients and controls, ${ }^{5,70}$ and AD patients and patients with other neuropsychiatric conditions. ${ }^{6,26,30,31,56}$ Cross-sectional studies using eye-tracker technology have also been conducted with young adults to assess cognitive function. ${ }^{60,61,76-78,88}$ The hippocampus ${ }^{34}$ and perirhinal cortex ${ }^{87}$ of monkeys have also been studied to address the role of different brain areas in memory tasks. Only two review studies have been found to address the use of eye movements to investigate the cognitive neuroscience of memory ${ }^{7}$ and the cognitive processes involved in smooth pursuit eye movements. ${ }^{51}$ Other studies involved vision and the visual system, as well as their impairments in AD. ${ }^{29,35-50,54}$ The lack of retrospective and prospective cohort studies indicates the need for further investigation to establish a direct relationship between eye movement patterns and the early stages of $\mathrm{AD}$, and also to cooperate to the development of a reliable tool to detect visual system abnormalities prior to cognitive and behavioral symptoms.

The development and origin of $\mathrm{AD}$ has been a recurrent theme among research communities, and a great deal of effort has been made in order to identify the earliest markers of the disease. A wide variety of biological, neurofunctional, and neuropsychological methods has been used to detect and predict the conversion of MCI to AD; however, this is a complex task given the lack of consensus around the definition and classification of MCI. In fact, if previous investigations considered this concept as a transitional state between normal aging and dementia, nowadays it can be considered as a clinical framework for itself with different subclassifications yet to be defined. Furthermore, increasing evidence indicates that episodic memory impairments might not be the only early changes that occur before conversion. Given the current debate about these different subtypes of MCI, it is relevant to consider every cognitive change that might be detectable at the early stages of the disease, since they can be considered valid predictive markers. Recent investigations have been indicating that other types of impairments can be present before establishing a diagnosis of $\mathrm{AD}$, namely in terms of visuospatial abilities and executive functioning.

Two of the most affected brain structures in AD pathology are the hippocampus and MTL structures, which are responsible not only for memory functioning, but also for visual processes. Furthermore, other impaired structures, such as the temporal and parietal lobes, are also associated with perceptual organization, shifts of attention, and spatial abilities. Given these reasons, it would be worthwhile to broaden the spectrum of the study of early cognitive markers in $\mathrm{AD}$ so it includes visual and executive functions. 
As mentioned previously, we presented several studies that confirmed the utility of eye movement behavior as a tool for identifying cognitive impairments in general, but also in specific pathological conditions, like AD. With respect to $\mathrm{AD}$, patients show different eye movement patterns, such as changes in fixation duration, saccade orientation, smooth pursuit movements, or saccadic inhibition, among others, even without participants' explicit reports during tasks. However, there is still insufficient investigation of these changes in MCI. Patients with this condition seem to show increased latencies in reflexive saccades and altered saccadic inhibition, which might suggest impairments in executive functioning. Nonetheless, the existing studies present reduced populations and a lack of follow-up information, especially with respect to the association between these eye movement changes and the conversion to $\mathrm{AD}$. Future investigations might be of benefit if they could integrate a time-course analysis to confirm the true effects of eye movement patterns in different subjects, so as to avoid overlooking these effects as single results. Moreover, the combination of eye movement techniques with neuroimaging and neuropsychological methods would help to improve the existing knowledge about the early impairments in $\mathrm{AD}$.

The study of eye movement behavior can also be a valuable tool for the assessment of early manifestations of memory impairments in dementia when combined with appropriate behavioral tasks that are specifically designed to highlight these deficits. Tasks like the visual paired-comparison task, sufficiently challenged visual recognition memory, so that $\mathrm{AD}$ patients can no longer distinguish between novel and previously seen items, suggesting that the same oculomotor pattern is evident for both of them. Moreover, in AD subjects, VSTM tasks can reveal deficits in the ability to bind different features of an item, and in item location in allocentric representations. Impairments in this ability to recollect, in an integrative way, associative information from memory are often related to early neuroanatomical changes, namely in hippocampus and entorhinal cortex, even in MCI patients. Therefore, we can assume that visual short-term binding deficits may be a preclinical marker of the disease. Recent investigations on VSTM deficits have been suggesting that iconic memory becomes impaired much faster in MCI patients compared to healthy controls; however, this is still an issue that receives little attention from researchers. It would be interesting to use eye movement analysis to further explore the more basic perceptive changes in VSTM, besides deficits in iconic memory in MCI/AD subjects, trying to unveil possible impairments in hippocampal functioning and the involvement of other cognitive domains. Also, the spatial nature of memory deficits in $\mathrm{AD} / \mathrm{MCI}$ seems to be a topic less explored by researchers, although the influence of the hippocampus and the temporoparietal network in this cognitive domain is generally known.

Another topic addressed in this review was that changes other than memory impairments might occur early in the course of AD - namely, in attention and executive functioning. $\mathrm{AD}$ patients showed alterations in the active shift of attention, which seems to grow as tasks demands are made more difficult. The majority of studies approaching attentional impairments fail to consider attention as a multidomain concept, and they seem to choose inadequate tasks that try to assess attention as a unique, global domain. ${ }^{80}$ Once again, there are few investigations around these deficits in MCI, but recent studies have been showing that this condition appears to exhibit impaired top-down processing. In addition, divided attention has been shown to be the most impaired subclassification in a hierarchical pyramid of attention deficits. These results still need to be corroborated by future studies, as they can be considered early signs of disease before the establishment of a diagnosis. In addition, AD patients also show changes in error correction saccades, and they exhibit a larger number of fixations with longer fixation duration as well. Some findings add that these alterations might be due to a deficit in disengaging visuospatial attention, while others attribute these changes to a failure to inhibit well-rehearsed strategies. ${ }^{78}$ In the future, more studies focusing on the neural correlates of oculomotor functions would be relevant, given that recent data indicated that there was a disruption of frontal activation, specifically in the frontal eye fields, in aMCI subjects who had registered an impairment in inhibitory functions. Nonetheless, we still need to understand whether this association between oculomotor behavior and neural correlates in aMCI patients may help to detect individuals who may convert to $\mathrm{AD}$.

In the Memory and attention expression in eye movement behavior: two systems operating together? section, we stressed the underlying difficulty in precisely discriminating the effects of memory and attention on visual processing, since some impairments in visual attention could be recast as impairments in VSTM and vice versa. This finding possibly reflects the double role played by some brain structures, such as the hippocampus and MTL, both in memory and in visual perception functions. In addition, some authors ${ }^{88}$ have argued that attention facilitates visuospatial memory for the relations between objects, characterized by the corresponding overt shifts of attention; others ${ }^{89}$ have claimed that deficits in visuospatial 
memory seem to be primary and reflect a genuine spatial disorder. Taking this into consideration, spatial impairments still need deeper scientific investigation, as they might appear earlier than other deficits in disease progression. Moreover, MCI patients seem to exhibit changes in visual processing in a more preattentive and earlier stage of the disease, preceding later impairments in VSTM. However, little is known about which MCI subtypes present with visual processing deficits that convert to $\mathrm{AD}$, and it is also unclear how these impairments progress during the course of the disease.

In sum, the debate around the early cognitive markers of AD pathology is still open, but we can assume that episodic memory deficits might not be the first ones to occur. Deficits of a more visual and executive nature might emerge earlier in the course of the disease. A combined effort between eye movement behavior and neuroimaging techniques with the appropriate neuropsychological tasks derived from cognitive psychology may reveal whether there is a relationship of coexistence or precedence between the different early signs of AD.

\section{Disclosure}

The authors report no conflicts of interest in this work.

\section{References}

1. Friedman NP, Miyake A, Young SE, Defries JC, Corley RP, Hewitt JK. Individual differences in executive functions are almost entirely genetic in origin. J Exp Psychol Gen. 2008;137(2):201-225.

2. Park DC, Reuter-Lorenz P. The adaptive brain: aging and neurocognitive scaffolding. Annu Rev Psychol. 2009;60:173-196.

3. Fox NC, Warrington EK, Seiffer AL, Agnew SK, Rossor MN. Presymptomatic cognitive deficits in individuals at risk of familial Alzheimer's disease. A longitudinal prospective study. Brain. 1998;121 (Pt 9):1631-1639.

4. Johnson DK, Storandt M, Morris JC, Galvin JE. Longitudinal study of the transition from healthy aging to Alzheimer disease. Arch Neurol. 2009;66(10):1254-1259.

5. Alichniewicz KK, Brunner F, Klünemann HH, Greenlee MW. Neural correlates of saccadic inhibition in healthy elderly and patients with amnestic mild cognitive impairment. Front Psychol. 2013;4:467.

6. Crutcher MD, Calhoun-Haney R, Manzanares CM, Lah JJ, Levey AI, Zola SM. Eye tracking during a visual paired comparison task as a predictor of early dementia. Am J Alzheimers Dis Other Demen. 2009;24(3):258-266.

7. Hannula DE, Althoff RR, Warren DE, Riggs L, Cohen NJ, Ryan JD. Worth a glance: using eye movements to investigate the cognitive neuroscience of memory. Front Hum Neurosci. 2010;4:166.

8. Lagun D, Manzanares C, Zola SM, Buffalo EA, Agichtein E. Detecting cognitive impairment by eye movement analysis using automatic classification algorithms. J Neurosci Methods. 2011;201(1): 196-203.

9. Petersen RC, Smith GE, Waring SC, Ivnik RJ, Tangalos EG, Kokmen E. Mild cognitive impairment: clinical characterization and outcome. Arch Neurol. 1999;56(3):303-308.

10. Petersen RC, Doody R, Kurz A, et al. Current concepts in mild cognitive impairment. Arch Neurol. 2001;58(12):1985-1992.

11. Petersen RC. Mild cognitive impairment as a diagnostic entity. J Intern Med. 2004;256(3):183-194.
12. Stephan BC, Hunter $\mathrm{S}$, Harris $\mathrm{D}$, et al. The neuropathological profile of mild cognitive impairment (MCI): a systematic review. Mol Psychiatry. 2012;17(11):1056-1076.

13. Galimberti D, Scarpini E. Progress in Alzheimer's disease. J Neurol. 2012;259(2):201-211.

14. Jack CR Jr, Weigand ST, Shiung MM, et al. Atrophy rates accelerate in amnestic mild cognitive impairment. Neurology. 2008;70(19 Pt 2): $1740-1752$

15. Dickerson BC, Sperling RA, Hyman BT, Albert MS, Blacker D. Clinical prediction of Alzheimer disease dementia across the spectrum of mild cognitive impairment. Arch Gen Psychiatry. 2007;64(12): $1443-1450$.

16. Drago V, Babiloni C, Bartrés-Faz D, et al. Disease tracking markers for Alzheimer's disease at the prodromal (MCI) stage. $J$ Alzheimers Dis. 2011;26 Suppl 3:159-199.

17. Dubois B, Feldman HH, Jacova C, et al. Revising the definition of Alzheimer's disease: a new lexicon. Lancet Neurol. 2010;9(11): $1118-1127$.

18. Mitchell AJ. Is it time to separate subjective cognitive complaints from the diagnosis of mild cognitive impairment? Age Ageing. 2008;37(5):497-499.

19. Palmer K, Fratiglioni L, Winblad B. What is mild cognitive impairment? Variations in definitions and evolution of nondemented persons with cognitive impairment. Acta Neurol Scand Suppl. 2003; 179:14-20.

20. Mitchell AJ, Shiri-Feshki M. Rate of progression of mild cognitive impairment to dementia - meta-analysis of 41 robust inception cohort studies. Acta Psychiatr Scand. 2009;119(4):252-265.

21. Hunderfund AL, Roberts RO, Slusser TC, et al. Mortality in amnestic mild cognitive impairment: a prospective community study. Neurology. 2006;67(10):1764-1768

22. Tales A, Snowden RJ, Haworth J, Wilcock G. Abnormal spatial and non-spatial cueing effects in mild cognitive impairment and Alzheimer's disease. Neurocase. 2005;11(1):85-92.

23. Rozzini L, Chilovi BV, Conti M, et al. Conversion of amnestic Mild Cognitive Impairment to dementia of Alzheimer type is independent to memory deterioration. Int $J$ Geriatr Psychiatry. 2007;22(12):1217-1222.

24. Bäckman L, Small BJ, Fratiglioni L. Stability of the preclinical episodic memory deficit in Alzheimer's disease. Brain. 2001;124(Pt 1): 96-102.

25. Spaan PE, Raaijmakers JG, Jonker C. Early assessment of dementia: the contribution of different memory components. Neuropsychology. 2005;19(5):629-640.

26. De Santi L, Lanzafame P, Spanò B, et al. Pursuit ocular movements in multiple sclerosis: a video-based eye-tracking study. Neurol Sci. 2011;32(1):67-71

27. Armstrong RA. Visual signs and symptoms in Parkinson's disease. Clin Exp Optom. 2008;91(2):129-138.

28. Armstrong RA. Alzheimer's disease and the eye. J Optom. 2009;2(3):103-111.

29. Rizzo M, Anderson SW, Dawson J, Nawrot M. Vision and cognition in Alzheimer's disease. Neuropsychologia. 2000;38(8): $1157-1169$.

30. Garbutt S, Matlin A, Hellmuth J, et al. Oculomotor function in frontotemporal lobar degeneration, related disorders and Alzheimer's disease. Brain. 2008;131(Pt 5):1268-1281.

31. Viskontas IV, Boxer AL, Fesenko J, et al. Visual search patterns in semantic dementia show paradoxical facilitation of binding processes. Neuropsychologia. 2011;49(3):468-478.

32. Daffner KR, Scinto LF, Weintraub S, Guinessey JE, Mesulam MM Diminished curiosity in patients with probable Alzheimer's disease as measured by exploratory eye movements. Neurology. 1992;42(2): $320-328$.

33. Daffner KR, Mesulam MM, Cohen LG, Scinto LF. Mechanisms underlying diminished novelty-seeking behavior in patients with probable Alzheimer's disease. Neuropsychiatry Neuropsychol Behav Neurol. 1999;12(1):58-66. 
34. Zola SM, Squire LR, Teng E, Stefanacci L, Buffalo EA, Clark RE. Impaired recognition memory in monkeys after damage limited to the hippocampal region. J Neurosci. 2000;20(1):451-463.

35. Cronin-Golomb A, Gilmore GC, Neargarder S, Morrison SR, Laudate TM. Enhanced stimulus strength improves visual cognition in aging and Alzheimer's disease. Cortex. 2007;43(7):952-966.

36. Cronin-Golomb A, Sugiura R, Corkin S, Growdon JH. Incomplete achromatopsia in Alzheimer's disease. Neurobiol Aging. 1993; 14(5):471-477.

37. Gilmore GC, Cronin-Golomb A, Neargarder SA, Morrison SR. Enhanced stimulus contrast normalizes visual processing of rapidly presented letters in Alzheimer's disease. Vision Res. 2005;45(8): 1013-1020.

38. Gilmore GC, Groth KE, Thomas CW. Stimulus contrast and word reading speed in Alzheimer's disease. Exp Aging Res. 2005;31(1): $15-33$.

39. Kurylo DD, Corkin S, Dolan RP, Rizzo JF 3rd, Parker SW, Growdon JH. Broad-band visual capacities are not selectively impaired in Alzheimer's disease. Neurobiol Aging. 1994;15(3):305-311.

40. Pache M, Smeets $\mathrm{CH}$, Gasio PF, et al. Colour vision deficiencies in Alzheimer's disease. Age Ageing. 2003;32(4):422-426.

41. Wijk H, Berg S, Sivik L, Steen B. Colour discrimination, colour naming and colour preferences among individuals with Alzheimer's disease. Int J Geriatr Psychiatry. 1999;14(12):1000-1005.

42. Arnold SE, Hyman BT, Flory J, Damasio AR, Van Hoesen GW. The topographical and neuroanatomical distribution of neurofibrillary tangles and neuritic plaques in the cerebral cortex of patients with Alzheimer's disease. Cereb Cortex. 1991;1(1):103-116.

43. Braak H, Braak E, Kalus P. Alzheimer's disease: areal and laminar pathology in the occipital isocortex. Acta Neuropathol. 1989;77(5): 494-506.

44. Lewis DA, Campbell MJ, Terry RD, Morrison JH. Laminar and regional distributions of neurofibrillary tangles and neuritic plaques in Alzheimer's disease: a quantitative study of visual and auditory cortices. J Neurosci. 1987;7(6):1799-1808.

45. McKee AC, Au R, Cabral HJ, et al. Visual association pathology in preclinical Alzheimer disease. J Neuropathol Exp Neurol. 2006;65(6):621-630.

46. Pearson RC, Esiri MM, Hiorns RW, Wilcock GK, Powell TP. Anatomical correlates of the distribution of the pathological changes in the neocortex in Alzheimer disease. Proc Natl Acad Sci U S A. 1985;82(13):4531-4534.

47. Gilmore GC, Levy JA. Spatial contrast sensitivity in Alzheimer's disease: a comparison of two methods. Optom Vis Sci. 1991;68(10):790-794.

48. Schlotterer G, Moscovitch M, Crapper-McLachlan D. Visual processing deficits as assessed by spatial frequency contrast sensitivity and backward masking in normal ageing and Alzheimer's disease. Brain. 1984;107(Pt 1):309-325.

49. Coyne AC, Liss L, Geckler C. The relationship between cognitive status and visual information processing. J Gerontol. 1984;39(6):711-717.

50. Nissen MJ, Corkin S, Buonanno FS, Growdon JH, Wray SH, Bauer J. Spatial vision in Alzheimer's disease. General findings and a case report. Arch Neurol. 1985;42(7):667-671.

51. Fukushima K, Fukushima J, Warabi T, Barnes GR. Cognitive processes involved in smooth pursuit eye movements: behavioral evidence, neural substrate and clinical correlation. Front Syst Neurosci. 2013;7:4.

52. Nakashima Y, Morita K, Ishii Y, Shouji Y, Uchimura N. Characteristics of exploratory eye movements in elderly people: possibility of early diagnosis of dementia. Psychogeriatrics. 2010;10(3):124-130.

53. Jones A, Friedland RP, Koss B, Stark L, Thompkins-Ober BA. Saccadic intrusions in Alzheimer-type dementia. J Neurol. 1983;229(3): 189-194.

54. Sadun AA, Borchert M, DeVita E, Hinton DR, Bassi CJ. Assessment of visual impairment in patients with Alzheimer's disease. Am J Ophthalmol. 1987;104(2):113-120.

55. Fletcher WA, Sharpe JA. Smooth pursuit dysfunction in Alzheimer's disease. Neurology. 1988;38(2):272-277.
56. Hutton JT, Nagel JA, Loewenson RB. Eye tracking dysfunction in Alzheimer-type dementia. Neurology. 1984;34(1):99-102.

57. Mosimann UP, Felblinger J, Ballinari P, Hess CW, Müri RM. Visual exploration behaviour during clock reading in Alzheimer's disease. Brain. 2004;127(Pt 2):431-438.

58. Müller G, Richter RA, Weisbrod S, Klingberg F. Impaired eye tracking performance in patients with presenile onset dementia. Int J Psychophysiol. 1991;11(2):167-177.

59. Yang Q, Wang T, Su N, Xiao S, Kapoula Z. Specific saccade deficits in patients with Alzheimer's disease at mild to moderate stage and in patients with amnestic mild cognitive impairment. Age (Dordr). 2013;35(4):1287-1298.

60. Hannula DE, Ryan JD, Tranel D, Cohen NJ. Rapid onset relational memory effects are evident in eye movement behavior, but not in hippocampal amnesia. J Cogn Neurosci. 2007;19(10):1690-1705.

61. Hollingworth A. Two forms of scene memory guide visual search: memory for scene context and memory for the binding of target object to scene location. Vis Cogn. 2009;17(1-2):273-291.

62. Grady CL, Craik FI. Changes in memory processing with age. Curr Opin Neurobiol. 2000;10(2):224-231.

63. Grady CL, McIntosh AR, Craik FI. Age-related differences in the functional connectivity of the hippocampus during memory encoding. Hippocampus. 2003;13(5):572-586.

64. Olson IR, Jiang Y. Is visual short-term memory object based? Rejection of the "strong-object" hypothesis. Percept Psychophys. 2002;64(7):1055-1067.

65. Parra MA, Abrahams S, Logie RH, Della Sala S. Visual short-term memory binding in Alzheimer's disease and depression. J Neurol. 2010;257(7):1160-1169.

66. Moscovitch M. The hippocampus as a "stupid," domain-specific module: implications for theories of recent and remote memory, and of imagination. Can J Exp Psychol. 2008;62(1):62-79.

67. Hassabis D, Maguire EA. Deconstructing episodic memory with construction. Trends Cogn Sci. 2007;11(7):299-306.

68. Troyer AK, Murphy KJ, Anderson ND, Hayman-Abello BA, Craik FI, Moscovitch M. Item and associative memory in amnestic mild cognitive impairment: performance on standardized memory tests. Neuropsychology. 2008;22(1):10-16.

69. Parra MA, Sala SD, Abrahams S, Logie RH, Méndez LG, Lopera F. Specific deficit of colour-colour short-term memory binding in sporadic and familial Alzheimer's disease. Neuropsychologia. 2011;49(7):1943-1952.

70. Loewenstein DA, Acevedo A, Luis C, Crum T, Barker WW, Duara R. Semantic interference deficits and the detection of mild Alzheimer's disease and mild cognitive impairment without dementia. J Int Neuropsychol Soc. 2004;10(1):91-100.

71. Lu ZL, Neuse J, Madigan S, Dosher BA. Fast decay of iconic memory in observers with mild cognitive impairments. Proc Natl Acad Sci US A. 2005;102(5):1797-1802.

72. Alescio-Lautier B, Michel BF, Herrera C, et al. Visual and visuospatial short-term memory in mild cognitive impairment and Alzheimer disease: role of attention. Neuropsychologia. 2007;45(8):1948-1960.

73. Schacter DL, editor. Memory Distortion: How Minds, Brains, and Societies Reconstruct the Past. Cambridge, MA: Harvard University Press; 1995.

74. Rosenbaum RS, Priselac S, Köhler S, et al. Remote spatial memory in an amnesic person with extensive bilateral hippocampal lesions. Nat Neurosci. 2000;3(10):1044-1048.

75. Hollingworth A, Williams CC, Henderson JM. To see and remember: visually specific information is retained in memory from previously attended objects in natural scenes. Psychon Bull Rev. 2001;8(4):761-768.

76. Henderson JM, Hollingworth A. Eye movements and visual memory: detecting changes to saccade targets in scenes. Percept Psychophys. 2003;65(1):58-71.

77. Ryan JD, Althoff RR, Whitlow S, Cohen NJ. Amnesia is a deficit in relational memory. Psychol Sci. 2000;11(6):454-461. 
78. Perry RJ, Hodges JR. Attention and executive deficits in Alzheimer's disease. A critical review. Brain. 1999;122(Pt 3):383-404.

79. Scinto LF, Daffner KR, Castro L, Weintraub S, Vavrik M, Mesulam MM. Impairment of spatially directed attention in patients with probable Alzheimer's disease as measured by eye movements. Arch Neurol. 1994;51(7):682-688.

80. Okonkwo OC, Wadley VG, Ball K, Vance DE, Crowe M. Dissociations in visual attention deficits among persons with mild cognitive impairment. Neuropsychol Dev Cogn B Aging Neuropsychol Cogn. 2008;15(4): 492-505.

81. Lonie JA, Tierney KM, Herrmann LL, et al. Dual task performance in early Alzheimer's disease, amnestic mild cognitive impairment and depression. Psychol Med. 2009;39(1):23-31.

82. Perry RJ, Hodges JR. Dissociation between top-down attentional control and the time course of visual attention as measured by attentional dwell time in patients with mild cognitive impairment. Eur J Neurosci. 2003; 18(2):221-226.

83. Rösler A, Mapstone ME, Hays AK, et al. Alterations of visual search strategy in Alzheimer's disease and aging. Neuropsychology. 2000; 14(3):398-408.

84. Crawford TJ, Higham S, Renvoize T, et al. Inhibitory control of saccadic eye movements and cognitive impairment in Alzheimer's disease. Biol Psychiatry. 2005;57(9):1052-1060.

85. Bélanger S, Belleville S, Gauthier S. Inhibition impairments in Alzheimer's disease, mild cognitive impairment and healthy aging: effect of congruency proportion in a Stroop task. Neuropsychologia. 2010;48(2):581-590.
86. Zhang Y, Han B, Verhaeghen P, Nilsson LG. Executive functioning in older adults with mild cognitive impairment: MCI has effects on planning, but not on inhibition. Neuropsychol Dev Cogn B Aging Neuropsychol Cogn. 2007;14(6):557-570.

87. Bussey TJ, Saksida LM, Murray EA. Perirhinal cortex resolves feature ambiguity in complex visual discriminations. Eur $J$ Neurosci. 2002;15(2):365-374.

88. Olsen RK, Chiew M, Buchsbaum BR, Ryan JD. The relationship between delay period eye movements and visuospatial memory. $J$ Vis. 2014;14(1). pii: 8.

89. Iachini I, Iavarone A, Senese VP, Ruotolo F, Ruggiero G. Visuospatial memory in healthy elderly, AD and MCI: a review. Curr Aging Sci. 2009;2(1):43-59.

90. Porter G, Leonards U, Wilcock G, Haworth J, Troscianko T, Tales A. New insights into feature and conjunction search: II. Evidence from Alzheimer's disease. Cortex. 2010;46(5):637-649.

91. Hort J, Laczó J, Vyhnálek M, Bojar M, Bures J, Vlcek K. Spatial navigation deficit in amnestic mild cognitive impairment. Proc Natl Acad Sci U S A. 2007;104(10):4042-4047.

92. Bundesen C. A theory of visual attention. Psychol Rev. 1990;97(4): 523-547.

93. Bublak P, Redel P, Sorg C, et al. Staged decline of visual processing capacity in mild cognitive impairment and Alzheimer's disease. Neurobiol Aging. 2011;32(7):1219-1230.
Neuropsychiatric Disease and Treatment

\section{Publish your work in this journal}

Neuropsychiatric Disease and Treatment is an international, peerreviewed journal of clinical therapeutics and pharmacology focusing on concise rapid reporting of clinical or pre-clinical studies on a range of neuropsychiatric and neurological disorders. This journal is indexed on PubMed Central, the 'PsycINFO' database and CAS,

\section{Dovepress}

and is the official journal of The International Neuropsychiatric Association (INA). The manuscript management system is completely online and includes a very quick and fair peer-review system, which is all easy to use. Visit http://www.dovepress.com/testimonials.php to read real quotes from published authors. 